\title{
Mechanobiological Assessment of TMJ Disc Surfaces: Nanoindentation and Transmission Electron Microscopy
}

\author{
Cassandra M. Juran'1, M. Franklin Dolwick², Peter S. McFetridge ${ }^{1}$ \\ ${ }^{1} \mathrm{~J}$. Crayton Pruitt Department of Biomedical Engineering, University of Florida, United States. \\ ${ }^{2}$ Division of Oral and Maxillofacial Surgery, College of Dentistry, University of Florida, United States.
}

\author{
Corresponding Author: \\ Peter S. McFetridge \\ J. Crayton Pruitt Family Department of Biomedical Engineering \\ University of Florida \\ Biomedical Sciences Building JG46, PO Box 116131, Gainesville, FL 32611- 6131 \\ United States \\ Phone: (352) 2739325 \\ Fax: (352) 3739221 \\ E-mail: pmcfetridge@bme.ufl.edu
}

\begin{abstract}
Objectives: Temporomandibular disc is a mechanically robust fibrocartilage tissue exhibiting highly elastic compressive, shear, and tensile moduli with structurally dense extracellular matrix that supports functional loading of the joint. The aim of this study was to illustrate structural complexities of the superior and inferior disc surfaces, to demonstrate the robust mechanical ability of the disc as a whole may be due to depth-dependent regional/layered variation, and also to provide characterization data imperative for future tissue engineering efforts focused on restoring function to the joint.

Material and Methods: Nanoindentation was used to assess tissue zones in conjunction with detailed Transmission Electron Microscopy to define structural attributes that influence the temporomandibular disc function.

Results: The disc architecture adjacent to the superior surface was shown to have three distinct regional segments within the interface layer: 1-a surface peripheral layer; 2-subsurface region; and 3-a layer of helical matrix bundles. The inferior surface displayed an interface layer $(20 \mu \mathrm{m})$ that showed limited cell populations with little depth-dependent structural variation, a stiffer elastic modulus and reduced energy dissipation compared to the superior surface. These data indicate that the primary function of the inferior surface is resistance to compression rather than load distribution during joint motion.

Conclusions: These are the first works that demonstrate that the superior central surface of the he temporomandibular disc is structured in depth-dependent isometric layers, each of which provides different mechanical function supporting the bulk tissue's properties. From a clinical perspective these data have potential to define regions susceptible to fatigue that may translate to diagnostic criteria to better define the stages of dysfunction.
\end{abstract}

Keywords: electrons; extracellular matrix; fibrocartilage; microscopy; temporomandibular joint; tissue engineering.

Accepted for publication: 14 December 2015

To cite this article:

Juran CM, Dolwick MF, McFetridge PS.

Mechanobiological Assessment of TMJ Disc Surfaces: Nanoindentation and Transmission Electron Microscopy

J Oral Maxillofac Res 2015;6(4):e3

URL: http://www.ejomr.org/JOMR/archives/2015/4/e3/v6n4e3.pdf

doi: $10.5037 /$ jomr.2015.6403 


\section{INTRODUCTION}

The temporomandibular joint (TMJ), the functional joint of the jaw, is arguably the most mechanically active and mechanically complex joint in the human body. The functional loading between the highly incongruent articulating skeletal structures is regulated and facilitated by the small fibrocartilage TMJ disc. Mechanical characterization of the disc has recently become an area of investigation and reports have shown the disc to be mechanically robust, exhibiting highly elastic compressive, shear, and tensile moduli. These properties are a consequence of the very dense and structurally organized extracellular matrix (ECM) adapted to support the repeated sliding action during functional loading [1-5].

Structural imaging has posited that the robust mechanical ability the disc possesses is due to the intricate architecture of the ECM fibrils, specifically collagen. Minarelli and Liberti [6] reported that the collagen fibril arrangement of the intermediate zone (central area of the disc) anterioposteriorly, mediolaterally and obliquely were responsible for the disc's role in resisting compression and stretching during occlusal loading. These observations were in disagreement with the works of Jagger [ $\underline{7}]$, where fibrils were observed only oriented in an anterior to posterior direction. De Bont et al. [ $\underline{8}]$, also observed that the articular surface of the disc was composed of densely packed collagen fibrils oriented anteroposteriorly with coiled fibrils at the most superficial layer of the surface. More recently Detamore et al. [ $[\underline{]}$, found that collagen fibres around the porcine TMJ disc have a ring like-structure around the periphery and are orientated anterior to posterior in the intermediate zones, agreeing with the work of Jagger [7] and other studies assessing human TMJ discs [9-13]. The porcine model has become prevalent in publications due to similarities of the joint and disc structure as well as physiologic action and chewing modalities to human TMJ structure/function $[13,14]$. However, previous investigations of the superior and inferior disc surfaces in relation to structure/function have focused on macro-scaled measurements where a more detail at the nano/micron level would aid further characterization and determine end points for disc reengineering.

These works hypothesize that the structural complexities seen in the imaging investigations and the robust mechanical ability of the TMJ disc may be due to regional or layered differences through the thickness of the intermediate surface zones, previously uncharacterized. Of significant importance for the purposes of these works will be the investigation of the TMJ disc's surface interface layers (both superior and inferior) by ultra nanoindentation in conjunction with detailed transmission electron microscopy (TEM). The interface layer is a layer of disc tissue immediately adjacent to the joint spaces directly influenced by articulation of the joint. The interface layer for other cartilaginous tissues in articulating joints is approximately $20-25 \mu \mathrm{m}$ [1ㄷ]. Thus nanoscale characterization of the surface interface layers using high resolution TEM and nanoindentation techniques will be applied to $20-30 \mu \mathrm{m}$ accordingly. These are the first investigations to use these evaluation techniques to assess the TMJ disc and as such provide unique insight into structure function relationships.

Micro- and nanoindentation techniques offer researchers unprecedented resolution of mechanical properties at the micron and nanometer scale. These techniques offer the advantages of sub-micron sized contact tips with spatial resolutions below $1 \mu \mathrm{m}$ and coupled with shallow indentation depths that provide a controllable approach to assess finer details of tissue structure. Gupta et al. [16], investigated the role of calcified cartilage within the human patella and how these zones influence load transfer from articular cartilage to subchondral bone. Nanoindentation results were correlated to mineral content within the various zones to provide a detailed material property map; information invaluable for further whole joint characterization. Zone specific micromechanical evaluation of human trabecular and compact bone by Zysset et al. [17] and growth plate cartilage by Radhakrishnan et al. [15] illustrated that these tissues could be described as an assembly of distinct micromechanical and structural units with seemingly homogeneous material properties.

Indentation properties of the TMJ disc's superior zone and the articular cartilage from the temporal fossa were compared by Kim et al. [18]. Findings were the articular cartilage had a $60 \%$ greater recovery compared to the disc, indicating cartilage associated with the temporal fossa plays a significant role in joint cushioning. More recently Yuya et al. [19], investigated anisotropy of mechanical ability of the TMJ disc respective of collagen fibre alignment. Results show that the response of the disc to indentation in the direction of collagen fibres effects a stiffer modulus of elasticity compared to indentation testing conducted obliquely to fibril alignment. A major difference between these seminal indentation studies by Kim et al. [18], or Yuya et al. [19], 
and these works is the indenter's geometry. Kim et al. [18] used a $2 \mathrm{~mm}$ diameter flattened indenter tip with a step resolution of $0.25 \mu \mathrm{m}$ and Yuya et al. [19] used a $50 \mu \mathrm{m}$ spherical indenter. These investigations use a CSM nanoindenter with a $10 \mu \mathrm{m}$ rounded tip, with a step resolution of $0.001 \mu \mathrm{m}$ and noise floor $\approx 1 \mathrm{~nm}$. The geometric properties of the nanoindenter offer higher resolution data collection, resulting in a more focused evaluation of the disc's micro architecture. This allows correlation of micro-architectural elements to depth dependent mechanical properties.

The aim of present study was to investigate the microscale mechanical ability of the disc surface and near surface regions using a nanoindentation approach and correlate the mechanical findings to the structural architecture illustrated by scanning and transmission electron microscopy.

\section{MATERIAL AND METHODS Tissue dissection and disc properties}

TMJs were harvested from 6 - 9 month old great white pigs by Animal Technologies (Tyler TX) with IACUC approval (IACUC Protocol \# 201207534). Within 24 hours, TMJ discs were isolated by separating the mandible from condyle and severing the connective tissues surrounding the disc. Immediately following dissection sampled discs were evaluated for central intermediate zone thickness. Discs where stored no longer than 6 hours in phosphate buffer saline (PBS $\mathrm{pH}$ 7.4) until further evaluation.

Independent TMJ disc samples were collected utilizing the same dissection method described to evaluate wet weight, dry weight and \%DNA/dry weight. Six separate TMJ central intermediate zone samples were isolated from the discs central zone using a $6 \mathrm{~mm}$ diameter biopsy punch and measured for wet weight. After wet weight measurement the samples were progressively frozen at $-20{ }^{\circ} \mathrm{C}$ for 6 hours followed by $-80{ }^{\circ} \mathrm{C}$ for a further 18 before lyophilization. After freezing, samples were lyophilized (freeze-dried) for 24 hours at $-84^{\circ} \mathrm{C}$ in vacuum less than $8 \mathrm{~m}$ Torr $(<1.66 \mathrm{~Pa})$ using a bench top freeze-drier (Millrock Technology, Kingston, NY). Upon completion the central disc zone punches' dry weights were measured. The dry samples were then digested using a papain solution to release DNA fragments from the cells for detection using QuantiiT Pico Green as per manufactures instructions (Invitrogen, Oregon, USA). Calibration curves were produced for known concentrations of cells to DNA that were then used to determine the DNA concentration/cell of $7.6 \mathrm{pg}$ DNA/cell.

\section{Depth dependent nanoindenter micromechanical testing}

Samples for mechanical evaluation were isolated from the discs central zone using a $6 \mathrm{~mm}$ diameter biopsy punch. The tissue punches were marked to ensure repeated sample loading orientation and tested immediately. Samples were immersed in a hydrated test chamber (PBS, pH 7.4) and oriented such that the anteroposterior fibre alignment was in the correct placement. All mechanical tests were performed using a CSM Instruments Ultra Nanoindentation Tester (UNTH, CSM VA) with a $10 \mu \mathrm{m}$ radius diamond spherical tip. Quasi-static and creep tests conducted on the superior and inferior surfaces were accomplished by loading the relative surface to $100 \mu \mathrm{N}$ holding for a 100 second creep analysis and unloading to measure recovery. Data was tabulated to report load versus depth and resulting stress/strain to determine compressive modulus of elasticity and \% hysteresis.

Progressive multi-cycle indentation was used to capture the change in mechanical properties versus indentation depth of the superior surface region. A 60 $\mu \mathrm{N}$ (starting load) to $200 \mu \mathrm{N}$ (finishing load) load was applied for each cycle to ensure uniform mechanical characterization at each depth zone. The sample was indented to $60 \mu \mathrm{N}$ and the indenter depth recorded, the indenter was then depressed until a $200 \mu \mathrm{N}$ load was reached, then maintained for 5 seconds. The sample was then unloaded and allowed to recover for 5 seconds before initializing the next loading cycle. Five indentation depths were used to evaluate depth-dependent mechanical characteristics with the indentation depth approximately $4.5 \mu \mathrm{m}$ per step. The force difference from start to final load per cycle was set at $140 \mu \mathrm{N}$ to maintain testing uniformity.

Data was processed to tabulate mechanical properties of clinical and research significance. The max load (FMAX), depth dependent load (F), indentation depth $(\mathrm{x})$ were experimentally measured and used to calculate contact stiffness $S=\frac{\Delta F}{\Delta x}$, contact depth $x_{C}=x-\epsilon \frac{F}{s}$, contact area $A_{C} \approx 2 \pi R_{i} h_{C}$. Tabulated indentation variables were used in the derivation of the indentation modulus $E^{*}=\frac{1}{2} \frac{\sqrt{\pi}}{\sqrt{A_{C}}} \frac{d F}{d x}$ $\underline{d F}$

with $\overline{d x}$ evaluated as the slope of the unloading curve.

\section{Scanning electron microscopy}

TMJ central intermediate zone biopsy samples isolated after tissue dissection were fixed in $2.5 \%$ 
glutaraldehyde in PBS overnight rinsed before secondarily fixation in osmium tetroxide $(1 \%$ in PBS). Samples were then dehydrated in graded ethanol rinses $(25,50,75,95$ and three times 100\%) before cryofracturing and critical point drying. Briefly, biopsy samples were inserted into Parafilm sleeves filled with $100 \%$ ethanol and placed in liquid nitrogen for approximately 120 seconds. Once frozen samples were cryofractured using a razor blade held at approximately $75^{\circ}$ angle to the superior surface and struck with a hammer. The fractured fragments were collected mounted and critically point dried. Before visualization samples were mounted and sputter coated with gold/palladium. Samples were examined using a Hitachi S-4000 FE SEM at $10 \mathrm{kV}$.

\section{Transmission electron microscopy}

Samples were fixed with Trumps, pH 7.2 (formalin, glutaraldehyde, and $0.2 \mathrm{M}$ sodium cacodylate buffer with sucrose) overnight at $4{ }^{\circ} \mathrm{C}$ and then repeatedly washed with $0.1 \mathrm{M}$ cacodylate buffer and $0.2 \mu \mathrm{m}$ filtered deionized water before dehydration by graded ethanol $(25,50,75,95$ and three times $100 \%)$ and infiltrate cold $100 \%$ ethanol LR white resin. Samples were then embedded in resin, cured at $60{ }^{\circ} \mathrm{C}$ for 72 hours then mounted for sectioning in the ultramicrotome $(\sim 70 \mathrm{~nm})$. Sections were collected and post stained with $2 \%$ (aq.) uranyl acetate 6 ' followed by Reynold's lead citrate 4' and examined on a Hitachi H-7000 at $100 \mathrm{kV}$ and imaged with Olympus Veleta camera and iTEM software (University of Florida ICBR, Gainesville FL).

\section{Statistical analysis}

Data for each test conducted was calculated based on testing of four samples $(n=4$, different source animals). Standard deviation and one-way analysis of variance (ANOVA) testing were calculated to determine statistical significance between data sets for biomechanics. The results were presented as mean and standard deviation (M [SD]). Significance was established using the Tukey-Kramer's Test $(\mathrm{P}=0.05$, $n=4)$.

\section{RESULTS}

The average thickness of the intermediate zone was $1.43(0.12) \mathrm{mm}$ with a corresponding mass of $3.36(0.21)$ g. SEM images in Figure 1 show a cross

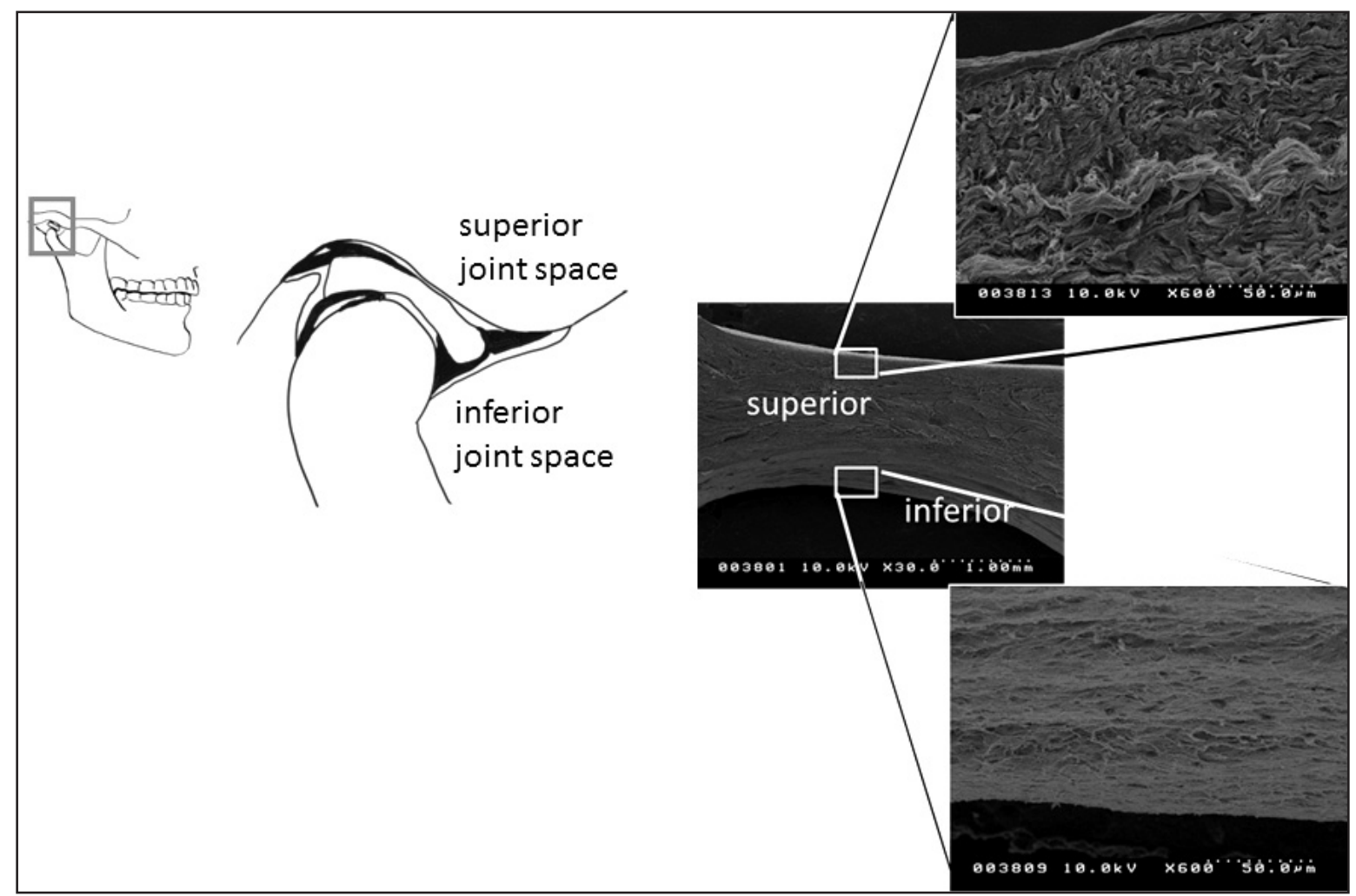

Figure 1. Joint and disc anatomy with depth dependent regional zone highlighted. Schematic joint anatomy with disc placement and orientation elucidated and SEM micrographs of the TMJ disc. The superior and inferior disc architecture has key differences which can be seen at low magnification and are accentuated at higher magnification. The superior disc surface is segmented into what appear to be several distinct surface regions before presenting the bulk architectural ECM matrix construction while the inferior surface appears to have one dense uniform surface region which appears more fibrous and linearized. 
section of the TMJ central zone at low magnification illustrating the biconcave ultrastructure of the disc. Higher magnifications of the surface zones show that each surface is structurally unique. SEM images of the superior surface suggests a layered structure with ECM fibrils most highly linearized and compacted at the joint interface and fibrils more undulant/ helical with distance from the surface. Relative to the superior surface, the inferior surface and underlying structures exhibited homogenous fibril structure with regular and linearized collagen bundles displaying a consistent porosity.

Nanoindentation step-creep comparison between the superior and inferior surface regions is shown in Table 1 and Figure 2 with corresponding high magnification TEM micrographs of the former and latter surfaces. The superior surface modulus is more elastic at $1.34 \mathrm{MPa}$ compared to the inferior at 2.49 MPa. Compressive elastic modulus data show the inferior zone to be $1.85 \mathrm{X}$ the stiffness of the superior surface. Additionally, the indentation modulus $E^{*}$ fits a liner curve for the inferior surface indicating uniform viscoelastic properties within the indented depths. Structurally Figure 2 TEM images illustrate the superior surface has fibril orientation anterioposteriorly and obliquely (arrows and crossed circle) while fibres on the inferior surface were comparatively isotropic, linearized and less compacted (arrows) with minimal cellularity (asterisks).

Table 1. Basic characterization of the porcine temporomandibular joint (TMJ) disc

\begin{tabular}{l|c|c}
\hline \multicolumn{1}{c|}{ TMJ properties } & Mean (SD) & Units \\
\hline Wet weight & $3(0.68)$ & $\mathrm{mg}$ \\
\hline Dry weight & $1.12(0.09)$ & $\mathrm{mg}$ \\
\hline Thickness (central/intermediate zone) & $1.97(0.21)$ & $\mathrm{mm}$ \\
\hline \%DNA content/weight & $0.16(0.01)$ & $\%$ \\
\hline
\end{tabular}

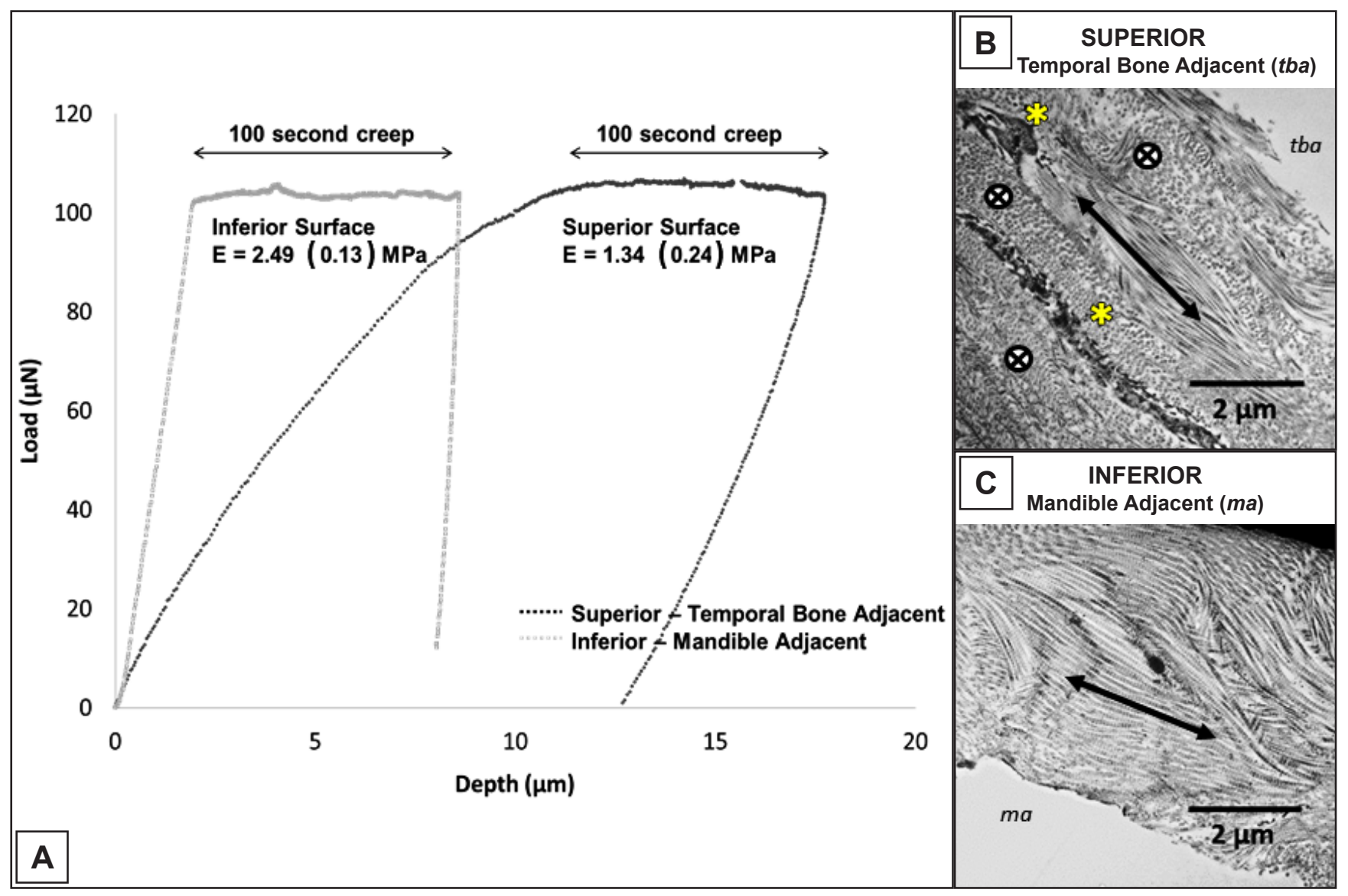

Figure 2. TEM micrographs and surface dependent step-creep response correlations. (A) illustrates a representative creep-relaxation indentation test. The inferior surface of the disc is mechanically stiffer and has less energy dissipation through indentation indicating that this side of the disc is primarily responsible for resistance to loading. The superior surface has a more elastic modulus and greater hysteresis indicating that this surface has more recoverability after loading. These mechanical findings are supported by high magnification TEM (B) and (C). (B) illustrates that the superior surface has fiber orientation both anterioposteriorly but also obliquely indicating that shear loading in multiple directions can be accommodated. The inferior surface (C) demonstrates linearized fibrils aligned anterior to posterior. These findings correlate with knowledge of joint function and architecture. The inferior disc surface does not have much relative motion to the mandible while the superior disc glides within the fossa and over the eminence of the temporal bone during function.

Arrowed lines indicate fibril alignment in anterior posterior direction, crossed circle indicate obliquely orientated fibrils, and asterisks identify cells. 


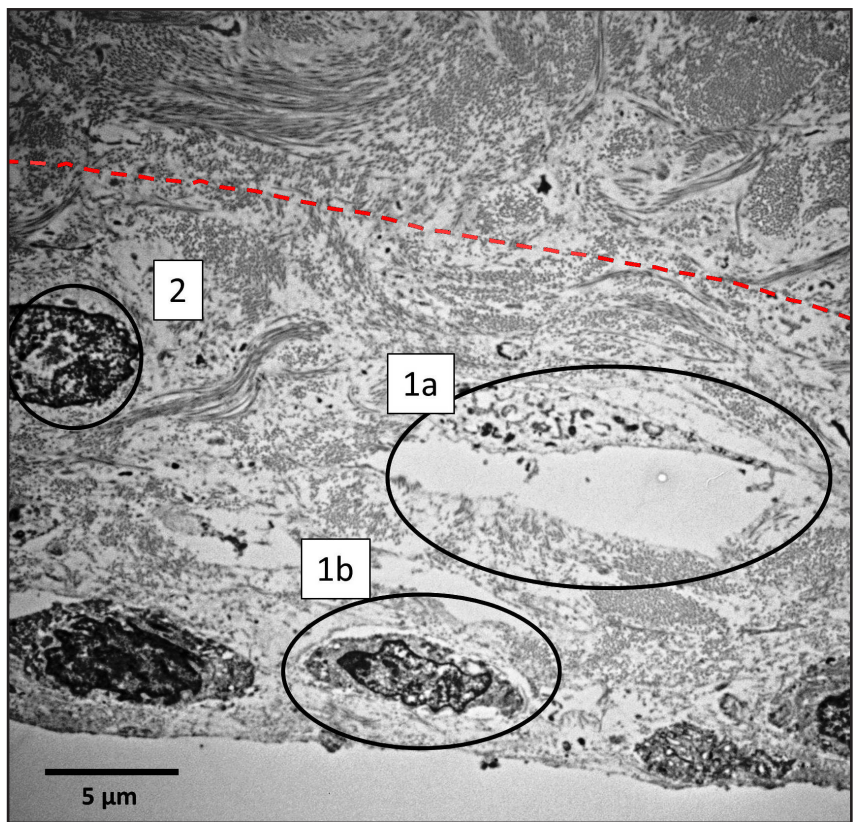

Figure 3. Apoptotic cell zone at inferior surface periphery. Imaged at the periphery of the inferior surface TEM slice this image shows apoptotic retraction of cells from the ECM (1a and 1b). Also lack of organelle structure is seen in necrotic cell (2) on path to apoptosis. All incidences occurred at the posterior periphery of the central intermediate zone. The dashed line indicates boundary of the inferior interface zone.

The TEM micrographs additionally demonstrate that the immediate $(<5 \mu \mathrm{m})$ structure from both the superior and inferior surface lack a cellular presence in the central region. The inferior disc surface zone (condyle side) has identifiable regions of apoptotic cell spaces as denoted by encirclement in Figure 3. These zones of apoptotic cell retraction from the ECM were found in 4 of the 6 imaged inferior sections, and all incidences occurred at the posterior periphery of the central intermediate zone. Visual identification of the apoptotic cells resulted in a ratio of 4 apoptotic or necrotic pre-apoptotic cells to every 6 normal matrix secreting cells.

Mechanical evaluation by multi-cyclic nanoindentation of the superior surface is depicted in Figure 4A and the resulting stress profiles and compressive modulus of elasticity shown in Figure $4 \mathrm{~B}$ and $4 \mathrm{C}$. The inferior surface was not evaluated utilizing this technique because results of the quasi-static creep analysis demonstrated the inferior surface's interface layer possessed uniform viscoelastic properties $\left(\mathrm{E}^{*}(x c)\right.$ values indicate no depth dependant characteristics within the evaluated interface layer) which was additionally supported by the high resolution TEM imaging of the inferior surface. Data shows the disc has a distinct surface zone of $4-6$ microns (pictorially loop a-b), which elicits the majority of the superior surface strength but has a reduced hysteresis of $31.37 \quad(3.37) \%$.

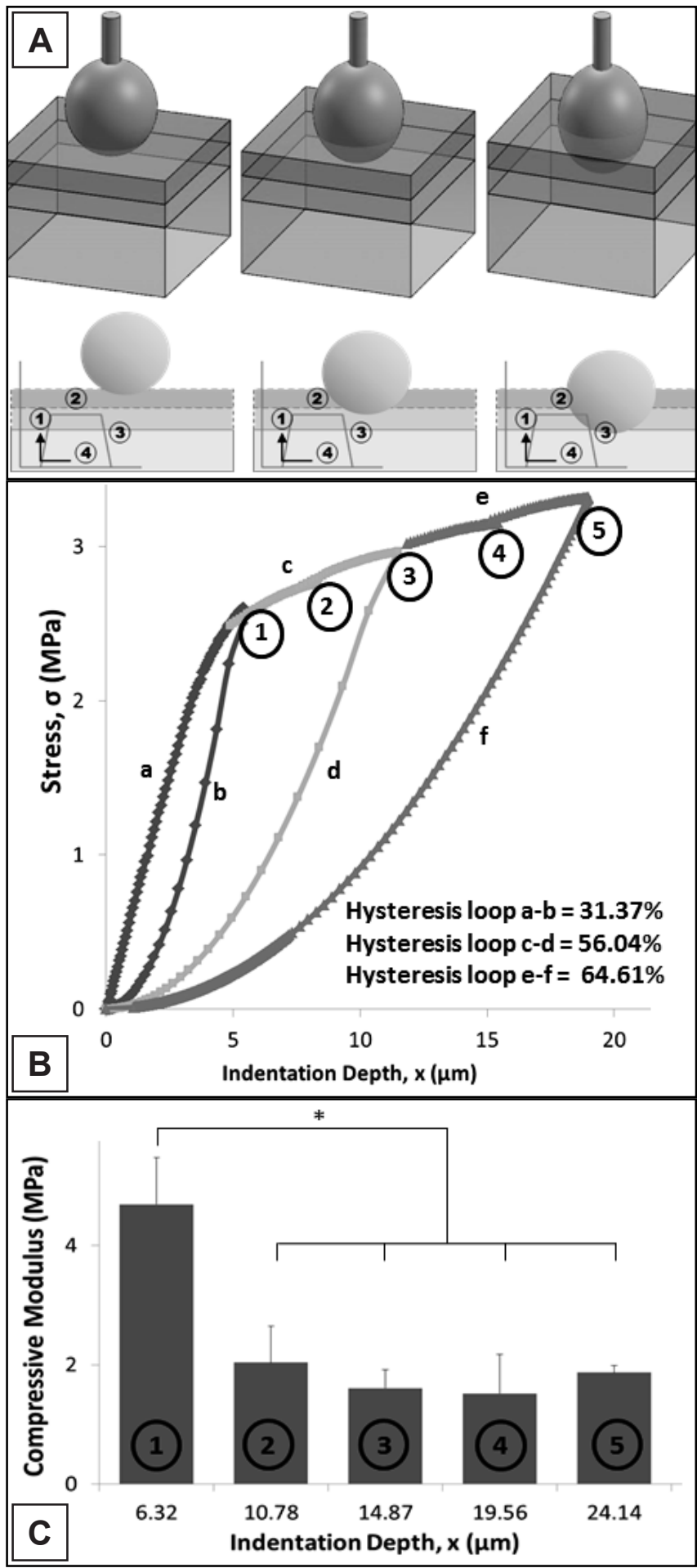

Figure 4. Cyclic ultra micro-indentation superior surface mechanical characterization. The micro-indentation methodology is illustrated in: (A) describing loading [1], holding [2], and unloading [3] then repeating for 5 cycles to obtain steady state mechanical properties [4] of the TMJ disc. Mechanical characterization of the surface, subsurface, and helical bundles layers are described in (B) and (C). Although five micro-indentation depths were tested (numbers 1 to 5 ) three distinct mechanical regions have been separated in (B) depicted by loops a-b, c-d, e-f, with loop a-b exhibiting the greatest resistivity to loading and loops c-d and e-f representing the surface regions with the greatest energy dispersion (hysteresis). The hysteresis loops correlate to the steady state compressive modulus of elasticity data shown in (C) $(\mathrm{n}=6, * \mathrm{P} \leq 0.01)$. 
Loops c-d, e-f and modulus values seen in Figure 4C (2 - 5) exhibit more elastic properties than the surface zone (loop a-b and modulus Figure 4C [1]).

TEM images of the superior surface (Figure 5A) indicate three structurally distinct layers: a highly compacted and anisotropic fibril orientation of the surface peripheral layer, a less compacted linearized and anterior to posterior orientated fibril subsurface layer containing greater cellular concentration and evidence of minor calcification, and a helical or bundular layer possessing few cells and an undulating fibril network. Magnifications of the three identified regions are detailed in Figure 5B. The surface peripheral layer denoted as segment- 1 is highly organized anterior to posterior (directionality indicated by arrow) with very compacted fibril matrix. This region greatly differs from segment-2 which was found to contain a large population of both fibroblast-like and chondrocyte-like cells (denoted by plus sign and asterisks respectively) and possess both anterior to posterior orientated and obliquely oriented ECM alignments. The third segment again possesses noteworthy differences from the previous described regions. Segment-3 presents highly crimped ECM structure with greater inter-bunduar spaces between structural fibrils.

\section{DISCUSSION}

The TMJ disc is positioned between the highly incongruent condyle and fossa skeletal structures. To facilitate mechanical loading the disc possesses a bi-concave structure located between superior and inferior synovial pockets. SEM examination of the disc's bi-concave structure demonstrates the two surfaces have markedly different ECM architecture. The tissue immediately beneath the disc's superior surface is arranged in layers that create depthdependent zones identifiable by scanning electron microscopy. Unlike the disc's superior surface the inferior surface is composed of well-defined linear fibril matrix with uniform porosity (Figure 1). This may indicate that this surface is less involved in recovery of the tissue after mechanical loading and acts more as an anchoring structure providing compressive resistance.

Creep response to ultra nanoindentation testing with a $100 \mu \mathrm{N}$ indentation load illustrates differences between the mechanical responsiveness of the superior and inferior surfaces. The inferior surface presents a resistive response to mechanical loading, with an elastic modulus of 2.5 MPa (Figure 2: mechanical assessment).

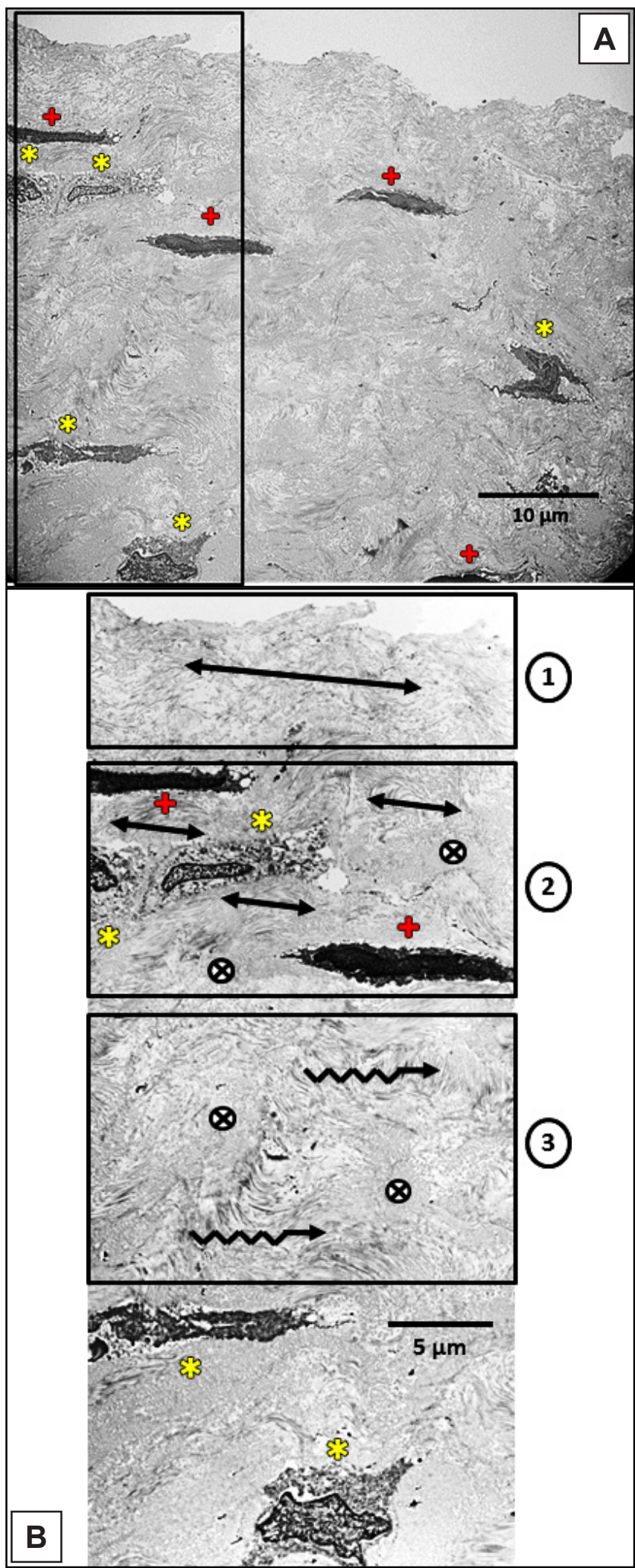

Figure 5. TEM of the superior disc surface: $A$ = illustrates that the disc architecture adjacent to the surface has three distinct regional segments: [1] a surface peripheral layer; [2] a subsurface region; and [3] a helical bundles layer. $\mathrm{B}=$ is a magnified selection of (A) more clearly denoting the three separable regions indicated by boxes [1] [2] and [3].

Arrowed lines indicate fibril alignment in anterior posterior direction, crossed circle indicate obliquely orientated fibrils, asterisks identify chondrocyte-like cells and plus sign denotes fibroblast-like cells. 
These findings correlate to the structural observations by TEM analysis (Figure 2: inferior TEM insert [arrows]) showing collagen fibres have a more linear orientation anterior to posterior. Limited cell populations were noted in the inferior TEM micrographs. This lack of cellular presence at the immediate inferior surface may be due to the smaller inferior synovial pocket and lesser degree of articulating motion between the disc and the condyle in comparison to the superior surface. Shi et al. [20], posited that mechanical movement encourages diffusive shuttling of nutrients through the disc. We hypothesize that comparatively to the discs superior counterpart the inferior interface layer's reduced mechanical movement in concert with a comparatively lesser nutrient source (synovial space and fluid volume) is the cause of the inferior interface layer's limited cell population and ineffective matrix recovery after joint loading. Micrographs of the peripheral inferior surface displayed remnants of dead cells (Figure 3) which supports our hypothesis that the inferior surface is not primarily responsible for the disc's ability to mechanically recover from loading but instead provides the robust resistance to compressive loading (stiffness) seen in mechanical evaluations of the whole disc. Several previous findings have demonstrated that cellular apoptosis and matrix degradation occur rapidly after traumatic injury within the condylar cartilage $[\underline{21}, \underline{22}]$, and that following injury chondrocytes from the proliferative zone differentiate and repopulate the incurred cellular void [21]. This mechanism found in the repeatedly loaded condylar cartilage provides further evidentiary support for our hypothesis that the TMJ disc is structured in distinct mechanical and functional layers to support function.

The superior surface of the disc demonstrates increased elastic qualities compared to the inferior surface (Figure 2: mechanical assessment) and greater hysteresis indicating that this surface has greater energy dissipative ability after loading. These mechanical findings are supported by high magnification TEM (Figure 2: superior TEM insert) showing that the superior surface has fibre orientation both anterioposteriorly but also obliquely. Indicating that shear loading in multiple directions can be accommodated at the superior disc's surface. Motion of the disc within the superior joint space is predominantly anteroposterior correlating to mouth opening and closing; however, the disc also translates mediolatterally with chewing and teeth grinding $[\underline{11}, \underline{23}]$. The multi-axial loading of the disc during jaw movement may be associated with the obliquely aligned collagen fibres observed in the superior surface micrograph that act to withstands these more variable physiological loading conditions.

To further investigate the complex architecture of the discs superior surface, ultra nanoindentation was applied to define depth-dependent mechanical regions. SEM was first used to broadly define structural layers to estimate indentations depths. Based on these estimations 5 indentation depths were tested with approximately $4.5 \mu \mathrm{m}$ separation. Mechanical stress as a function of indentation depth (Figure 4B), shows that between each of the 5 indentation depths (labeled $1-5)$ three mechanically distinct layers exist (labelled as loops). The layer with the highest rigidity and the most resistance to mechanical strain was shown to be immediately adjacent to the surface (loop a-b). This layer, designated as a surface peripheral layer, has little energy recuperative ability with a \% hysteresis of 31.37 (3.37)\%, with a linearized network of ECM fibres orientated primarily anterior-posterior (Figure 5B: regional segment 1). While not evaluated in these works we have previously evaluated the shear properties of the disc showing that the center zone of the disc is responsible for the majority of the shear strength in the disc [4]. In concert with this previous data we hypothesized that this surface peripheral layer (4.5 - $6.5 \mu \mathrm{m}$ depth) is primarily supportive of the gliding/sliding mechanism of TMJ disc over the articulation fossa of the temporal bone rather than compressive resistance.

Cyclic compressive loading at indentation depths 2 and $3(8.5-13.5 \mu \mathrm{m}$ depths $)$ produce a stress curve with similar slopes, indicating that these two indentation depths are within the same structural layer. This subsurface layer offers the least compressive resistance eliciting a decreased compressive elastic modulus of 1.98 (0.43) MPa. TEM imaging indicates that these mechanical properties corresponding to regional segment 2 presents higher cell concentrations than the surface peripheral layer where few cells were observed. This is likely the region responsible for cellular regeneration after disc surface damage that may result from impact, dysfunction, or disease.

Figure 5: regional segment 3 TEM illustrates a near acellular region with an anterior-posteriorly orientated undulating fibril structure, similar to observations from de Bont's group []. This observation was consistent for all sectional evaluated with an average 1 cell observation every $80 \mu \mathrm{m}$ imaged. The depths associated with these structural observation correlate to indentation depths 4 and 5, with similar mechanical characteristics showing compressive modulus properties exhibiting greater stiffness's at depths $2 / 3$ but more elastic qualities than depth 1 . 
The helical bundles presented in this layer have an elastic modulus comparative to the surface peripheral layer, having the highest \% hysteresis of all layers. The greater energy dissipation (64.61 [2.44]\%) within this layer is likely due to the crimped architecture of the collagen bundles that linearize to absorb loading stresses. Then as the load is removed the bundles regain their original crimped conformation ready to absorb the next load. From these mechanical findings we hypothesize this is the major structural supportive layer of the superior disc surface regions acting to support resistance to compression and functions to absorb and disseminate repetitive loading without structural impairment.

Calcification of the TMJ disc has been noted both in clinical and characterization publications $[24,25]$. These calcification events are generally considered a secondary effect of synovial chondromatosis [26], however evidence of calcification penetrating as far as $0.25 \mathrm{~mm}$ from the superior surface complicates this explanation. Experimentation has shown that matrix mineralization occurs in hypertrophic chondrocyte cultures and increased mechanical stimulation encourages pre-hypertrophic behavior in growth plate cartilage cells [27]. With this in mind a potential link between TMJ disc mineralization and the depth dependent layered structure of the TMJ can be explored. Mechanical characteristics (Figure 4) correlated with visual TEM definition (Figure 5) of the superior surface demonstrates that the structural layer with the largest cell population corresponds to a region not mechanically optimal for energy dissipation.

During normal joint motion, we have shown the interface surface layer (Figure 5B: regional segment 1) is the principal anatomical layer responsible for mechanical resilience to repeated jaw motion having the most rigidity (lowest \% hysteresis). Under pathogenic conditions where this layer is disturbed or destroyed the significantly weaker subsurface layer (Figure 5B: regional segment 2) is directly exposed to these repeated loads. These cells now exposed to pathologic mechanical stimulation have the potential to become pre-hypertrophic and secrete mineral matrix, as seen in growth plate cartilage ossification. With repeated loading and no regenerative action being undertaken by the subsurface layer cells continued calcification would develop calcium nodes seen in some early displacement patients. These findings suggest that clinical therapy to reduce pathologic loading on the disc in early stage TMDs may be of critical importance of patient recovery.

\section{CONCLUSIONS}

These findings derived through the use of nanoindentation techniques further our understanding of the finer detail of the mechanical architecture of articulating tissue. These works demonstrated that the central zone of the temporomandibular disc is structured in isometric depth-dependent layers which provide different mechanical properties that impart normal physiological function. The inferior surface (interfacial) has a minimal cellular composition with little structural variation and offers comparatively less energy dissipation in comparison to the superior surface. The superior surface presents three distinct mechanical and structural layers each responsible for different mechanical characteristics supporting the action of articulation within the temporomandibular joint. Clinically these data have the potential to define regions of the disc more susceptible to mechanical fatigue that may translate to diagnostic criteria that better defines stages of dysfunction. Many temporomandibular joint disorders are detected after significant damage to the underlying structure of the disc has occurred. Knowing the mechanical and visual qualities of the healthy disc will provide a basis for diagnostic imaging and minimally intrusive investigation of new patients. High resolution imaging during arthroscopic investigation of the joint may detect anomalies in the interface layer's texture before the disorder progresses allowing for early stage treatment if needed. Future diagnostic relevance may incorporate measurement of the interface layer's mechanical properties into arthroscopic evaluation as many robotic arthroscopes already use a small load cell to assess interaction with the tissue surface. This type of application would be a natural segue from existing treatment technologies for diagnostic purposes. In addition, micro and nano scaled characterization is imperative for further advances in the field of functionalized tissue engineering where the importance of interfacial mechanobiology is critical in governing whole tissue integration, adaptation, and regeneration.

\section{ACKNOWLEDGMENTS AND DISCLOSURE STATEMENT}

We gratefully acknowledge Dr. Bo Zhou of CSM Instruments (Boston, MA, USA) for the collection of the nanoindentation data. Also the National Institute of Health (NIH) National Institute of Dental and Craniofacial Research (1R21DE022449) for funding. This work is free of any conflict of interest. 


\section{REFERENCES}

1. Tanaka E, van Eijden T. Biomechanical behavior of the temporomandibular joint disc. Critical Reviews in Oral Biology \& Medicine. 2003 May; 14(2):138-150. [Medline: 12764076] [doi: 10.1177/154411130301400207]

2. Lumpkins $\mathrm{S}, \mathrm{McF}$ etridge P. Regional variations in the viscoelastic compressive properties of the temporomandibular joint disc and implications toward tissue engineering. J Biomed Mater Res A. 2009 Sep 1;90(3):784-91. [Medline: 18615466] [doi: 10.1002/jbm.a.32148]

3. Kuo J, Zhang L, Bacroc T, Yao H. The region-dependent biphasic viscoelastic properties of human temporomandibular joint discs under confined compression. J Biomech. 2010 May 7;43(7):1316-21. [Medline: 20171639] [doi: 10.1016/i.jbiomech.2010.01.020]

4. Juran C, Dolwick M, McFetridge P. Shear Mechanics of the TMJ Disc: Relationship to Common Clinical Observations. J Dent Res. 2013 Feb;92(2):193-8. [Medline: 23166043] [doi: 10.1177/0022034512468749]

5. Detamore M, Athanasiou K. Tensile properties of the porcine temporomandibular joint disc. J Biomech Eng. 2003 Aug;125(4):558-65. [Medline: 12968581] [doi: 10.1115/1.1589778]

6. Minarelli A, Liberti E. A microscopic survey of the human temporomandibular joint disc. J Oral Rehabil. 1997 Nov;24(11):835-40. [Medline: 9426165] [doi: 10.1111/j.1365-2842.1997.tb00283.x]

7. Jagger R. The surface-structure of the temporomandibular-joint disk - a scanning electron-microscopic study. J Oral Rehabil. 1980 May;7(3):225-34. [Medline: 6931198] [doi: 10.1111/j.1365-2842.1980.tb00439.x]

8. De Bont L, Liem R, Havinga P, Boering G. Fibrous component of the temporomandibular joint disk. Cranio. 1985 SepDec;3(4):368-73. [Medline: 3864873]

9. Nickel J, Mclachlan K. An analysis of surface congruity in the growing human temporomandibular-joint. Arch Oral Biol. 1994 Apr;39(4):315-21. [Medline: 8024496] [doi: 10.1016/0003-9969(94)90123-6]

10. Piette E. Anatomy of the human temporomandibular joint. An updated comprehensive review. Acta Stomatol Belg. 1993 Jun;90(2):103-27. [Medline: 8237635]

11. Naeije M, Hofman N. Biomechanics of the human temporomandibular joint during chewing. J Dent Res. 2003 Jul;82(7):528-31. [Medline: 12821713] [doi: 10.1177/154405910308200708]

12. Johns D, Athanasiou K. Design characteristics for temporomandibular joint disc tissue engineering: learning from tendon and articular cartilage. Proc Inst Mech Eng H. 2007 Jul;221(5):509-26. [Medline: 17822153] [doi: 10.1243/09544119JEIM158]

13. Bermejo A, Gonzalez O, Gonzalez J. The pig as an animal-model for experimentation on the temporomandibular articular complex. Oral Surg Oral Med Oral Pathol. 1993 Jan;75(1):18-23. [Medline: $\underline{8419867]}$ [doi: 10.1016/0030-4220(93)90399-O]

14. Kalpakci K, Willard V, Wong M, Athanasiou K. An Interspecies Comparison of the Temporomandibular Joint Disc. J Dent Res. 2011 Feb;90(2):193-8. [Medline: 21118792] [doi: 10.1177/0022034510381501]

15. Radhakrishnan P, Lewis N, Mao J. Zone-specific micromechanical properties of the extracellular matrices of growth plate cartilage. Ann Biomed Eng. 2004 Feb;32(2):284-91. [Medline: 15008376] [doi: 10.1023/B:ABME.0000012748.41851.b4]

16. Gupta H, Schratter S, Tesch W, Roschger P, Berzlanovich A, Schoeberl T, Klaushofer K, Fratzl P. Two different correlations between nanoindentation modulus and mineral content in the bone-cartilage interface. J Struct Biol. 2005 Feb;149(2):138-48. [Medline: $\underline{15681230]}$ [doi: 10.1016/j.jsb.2004.10.010]

17. Zysset P, Kulik A, Hengsberger S. A combined atomic force and nanoindentation technique to investigate the elastic properties of bone structural units. Eur Cell Mater. 2001 Jan 10;1:12-7. [Medline: 14562266]

18. Kim K, Wong M, Helfrick J, Thomas J, Athanasiou K. Biomechanical tissue characterization of the superior joint space of the porcine temporomandibular joint. Ann Biomed Eng. 2003 Sep;31(8):924-30. [Medline: 12918907] [doi: $10.1114 / 1.1591190]$

19. Yuya P, Amborn E, Beatty M, Turner J. Evaluating Anisotropic Properties in the Porcine Temporomandibular Joint Disc Using Nanoindentation. Ann Biomed Eng. 2010 Jul;38(7):2428-37. [Medline: 20195763] [doi: $10.1007 / \mathrm{s} 10439-010-9967-8$ ]

20. Shi C, Wright G, Ex-Lubeskie C, Bradshaw A, Yao H. Relationship between anisotropic diffusion properties and tissue morphology in porcine TMJ disc. Osteoarthritis Cartilage. 2013 Apr;21(4):625-33. [Medline: 23353670] [doi: 10.1016/i.joca.2013.01.010]

21. Li L, Wang L, Sun Y, Yang C, He D. Establishment and Histological Evaluation of a Goat Traumatic Temporomandibular Joint Model. J Oral Maxillofac Surg. 2015 May;73(5):943-50. [Medline: 25883002] [doi: 10.1016/j.joms.2014.12.038]

22. Embree M, Iwaoka G, Kong D, Martin B, Patel R, Lee A, Nathan J, Eisig S, Safarov A, Koslovsky D. Soft tissue ossification and condylar cartilage degeneration following TMJ disc perforation in a rabbit pilot study. Osteoarthritis Cartilage. 2015 Apr;23(4):629-39. [Medline: 25573797] [doi: 10.1016/j.joca.2014.12.015]

23. Wilding R, Lewin A. A computer-analysis of normal human masticatory movements recorded with a sirognathograph. Arch Oral Biol. 1991;36(1):65-75. [Medline: 2012528] [doi: 10.1016/0003-9969(91)90055-Y] 
24. Jibiki M, Shimoda S, Nakagawa Y, Kawasaki K, Asada K, Ishibashi K. Calcification of the disc of the temporomandibular joint. J Oral Pathol Med. 1999 Oct;28(9):413-9. [Medline: 10535365] [doi: 10.1111/j.1600-0714.1999.tb02113.x]

25. Mercuri L, Ali F, Woolson R. Outcomes of total alloplastic replacement with periarticular autogenous fat grafting for management of reankylosis of the temporomandibular joint. J Oral Maxillofac Surg. 2008 Sep;66(9):1794-803. [Medline: 18718385] [doi: 10.1016/j.joms.2008.04.004]

26. Koyama J, Ito J, Hayashi T, Kobayashi F. Synovial chondromatosis in the temporomandibular joint complicated by displacement and calcification of the articular disk: report of two cases. AJNRAm J Neuroradiol. 2001 Jun-Jul;22(6):1203-6. [Medline: 11415920]

27. Coe M, Summers T, Parsons S, Boskey A, Balian G. Matrix mineralization in hypertrophic chondrocyte cultures. Beta glycerophosphate increases type X collagen messenger RNA and the specific activity of pp60c-src kinase. Bone Miner. 1992 Aug;18(2):91-106. [Medline: 1381978] [doi: 10.1016/0169-6009(92)90850-D]

\section{To cite this article:}

Juran CM, Dolwick MF, McFetridge PS.

Mechanobiological Assessment of TMJ Disc Surfaces: Nanoindentation and Transmission Electron Microscopy

J Oral Maxillofac Res 2015;6(4):e3

URL: http://www.ejomr.org/JOMR/archives/2015/4/e3/v6n4e3.pdf

doi: $\underline{10.5037 / j o m r .2015 .6403}$

Copyright (C) Juran CM, Dolwick MF, McFetridge PS. Published in the JOURNAL OF ORAL \& MAXILLOFACIAL RESEARCH (http://www.ejomr.org), 31 December 2015.

This is an open-access article, first published in the JOURNAL OF ORAL \& MAXILLOFACIAL RESEARCH, distributed under the terms of the Creative Commons Attribution-Noncommercial-No Derivative Works 3.0 Unported License, which permits unrestricted non-commercial use, distribution, and reproduction in any medium, provided the original work and is properly cited. The copyright, license information and link to the original publication on (http://www.ejomr.org) must be included. 